\title{
Avaliação cefalométrica da estabilidade pós-expansão rápida da maxila assistida cirurgicamente*
}

Pedro Luis Scattaregi**, Danilo Furquim Siqueira***

\section{Resumo}

Objetivo: avaliar as possíveis alterações e a estabilidade dentária e esquelética no sentido transversal, bem como as possíveis alterações verticais da face (AFAI), produzidas pela Expansão Rápida da Maxila Assistida Cirurgicamente (ERMAC). Métodos: a amostra selecionada para este estudo retrospectivo foi composta por 60 telerradiografias em norma frontal, de 15 pacientes, sendo 6 do gênero masculino e 9 do gênero feminino, com média de idades de 23 anos e 3 meses. O disjuntor Hyrax foi instalado e o procedimento cirúrgico adotado envolveu a separação da sutura palatina mediana e não-abordagem da sutura pterigomaxilar. A ativação foi realizada do terceiro dias após a cirurgia até o término da expansão, determinada por critérios clínicos. Todos os pacientes foram radiografados nas fases pré-expansão (T1); pós-expansão imediata (T2); 3 meses pós-expansão, com o próprio disjuntor como contenção (T3); e 6 meses pós-expansão, com a placa removível de acrílico como contenção (T4). Medidas lineares foram obtidas a partir dos traçados cefalométricos gerados por um programa computadorizado (Radiocef Studio 2) e analisadas estatisticamente pelos testes de variância (ANOVA) e Tukey ao nível de 5\% de significância. Resultados e Conclusões: concluiu-se que a ERMAC produziu aumentos estatisticamente significativos da cavidade nasal, da largura maxilar e da distância intermolares superiores, de T1 para T2, os quais se mantiveram em T3 e T4. A largura facial e as distâncias intermolares inferiores não apresentaram alterações após a ERMAC. Avaliando o comportamento vertical da face, notou-se um aumento da AFAI nas fases T1 para T2, que diminuiu após a contenção de 3 meses (T3) e permaneceu estável em T4, embora aumentada se comparada com T1.

Palavras-chave: Expansão maxilar. Cirurgia maxilofacial. Cefalometria. 


\section{INTRODUÇÃO}

O tratamento ortodôntico tem por finalidade promover a correção da má oclusão, redirecionar o crescimento do complexo craniofacial, restabelecer a harmonia e a estética facial, bem como manter o equilíbrio do sistema estomatognático. Para isso, exames complementares - como fotografias, modelos, radiografias e análises cefalométricas - tornam-se imprescindíveis para estabelecer um bom diagnóstico e plano de tratamento, na busca incessante por resultados adequados e com estabilidade.

A deficiência transversal da maxila - dentre as várias formas de manifestação das más oclusões anteroposteriores, verticais ou transversais - está muito presente na população que busca o tratamento ortodôntico. Um sinal clínico da falta de crescimento e desenvolvimento transversal consiste na presença da mordida cruzada posterior uni ou bilateral. A mordida cruzada posterior pode ser corrigida com sucesso, durante a fase de crescimento, com recursos que proporcionam alterações dentoalveolares ou ortopédicas. A Expansão Rápida da Maxila (ERM), citada pela primeira vez na literatura por Angell1,2 em 1860 e reintroduzida por $\mathrm{Haas}^{17}$ em 1961, é um recurso incorporado ao tratamento ortodôntico que proporciona o ajuste transversal do arco dentário superior por meio da separação da sutura palatina mediana.

A ERM pode tratar a deficiência transversal da maxila com maior estabilidade em longo prazo, melhor estética dentofacial e saúde periodontal, dependendo das forças utilizadas e do grau de maturidade óssea ${ }^{5,23}$. Já em pacientes adultos, a maturidade esquelética alcança estágios de resistência praticamente impossíveis de serem vencidos pela ação ortopédica, exigindo, muitas vezes, um procedimento cirúrgico. Nesses casos, a Expansão Rápida da Maxila Assistida Cirurgicamente (ERMAC) deve ser indicada por meio de osteotomias em áreas de maior resistência da maxila, principalmente o complexo nasomaxilar e as suturas palatina mediana, zigomatomaxilar e pterigopalatina $^{9,10,17,20,23}$.

Estudos demonstraram que as alterações anteroposteriores e verticais promovidas pela ERMAC são interpretadas com maior facilidade por meio de telerradiografias em norma lateral ${ }^{31}$. Entretanto, para avaliar as alterações transversais, o exame complementar indicado é a telerradiografia em norma frontal ${ }^{5,17,21,26-29}$.

Segundo Proffit, Turvey e Phillips ${ }^{25}$, a ERMAC é um dos procedimentos cirúrgicos mais instáveis, ao se considerar a escala da hierarquia de estabilidade das cirurgias craniofaciais. Em razão da pequena literatura encontrada, o presente estudo propôs-se a avaliar a estabilidade das dimensões transversais dentárias e esqueléticas, bem como a altura facial anteroinferior, nas telerradiografias em norma frontal imediatamente após a ERMAC e decorridos três e seis meses de estabilização do parafuso expansor.

\section{MATERIAL E MÉTODOS Material}

A amostra selecionada para esse estudo retrospectivo foi composta por 60 telerradiografias em norma frontal, de 15 pacientes, sendo 6 do gênero masculino e 9 do gênero feminino, com média de idades de 23 anos e 3 meses, variando de 18 a 35 anos. Todos os pacientes foram submetidos à ERMAC e pertenciam aos arquivos do Programa de Pós-graduação em Odontologia - área de concentração Ortodontia - da Universidade Metodista de São Paulo (Umesp) e também ao Serviço de Cirurgia Craniomaxilofacial e Divisão de Cirurgia Plástica e Queimaduras do Instituto Central do Hospital das Clínicas da Faculdade de Medicina da Universidade de São Paulo (HCFMUSP). Os critérios de seleção dos pacientes foram ${ }^{14}$ : adultos com maturação esquelética definida; presença da atresia maxilar de $5 \mathrm{~mm}$ ou mais ${ }^{5}$; mordida cruzada posterior uni ou bilateral; sem tratamento ortodôntico prévio e ausência de síndromes craniofaciais. 


\section{Métodos}

\section{Documentação ortodôntica}

Para esse estudo foram utilizadas apenas as telerradiografias em norma frontal, embora os pacientes tenham feito outros exames complementares que compuseram as documentações ortodônticas.

As telerradiografias utilizadas para a comparação foram em número de quatro, todas em norma frontal (posteroanterior), para cada paciente selecionado, realizadas ao início do tratamento, na fase pré-expansão (T1); imediatamente após a expansão rápida da maxila assistida cirurgicamente (T2); aos três meses pós-expansão, com o próprio aparelho expansor como contenção (T3); e aos seis meses pós-expansão, com placa removível de acrílico como contenção (T4) - totalizando 60 telerradiografias (Fig. 1).
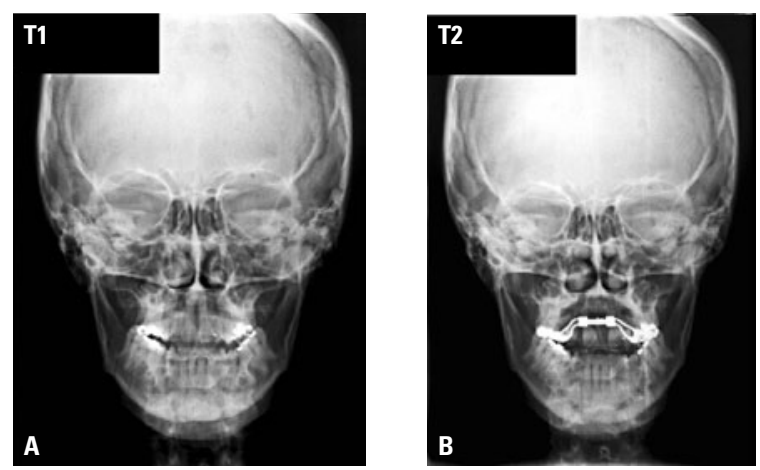

FIGURA 1 - Telerradiografias em norma frontal: A) pré-expansão (T1), B) imediatamente após a expansão (T2), C) três meses pós-expansão (T3) e D) seis meses pós-expansão (T4).

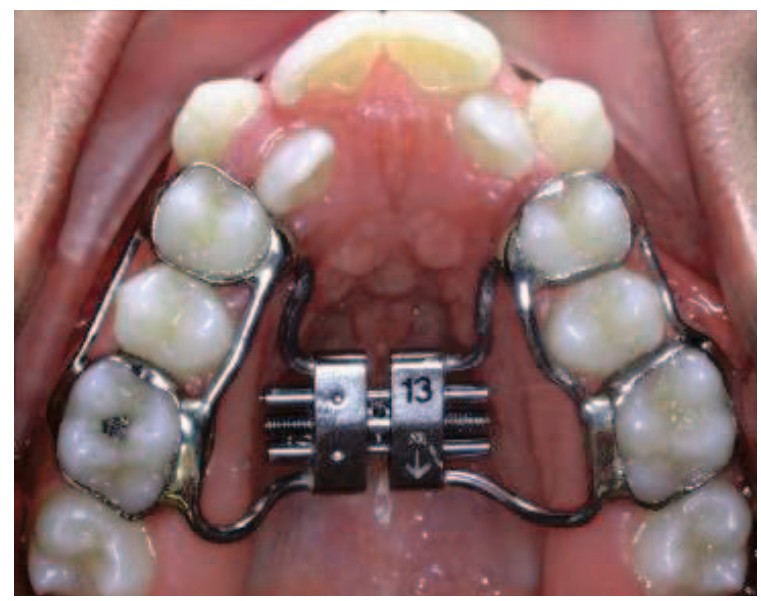

FIGURA 2 - Aparelho expansor fixo tipo Hyrax.
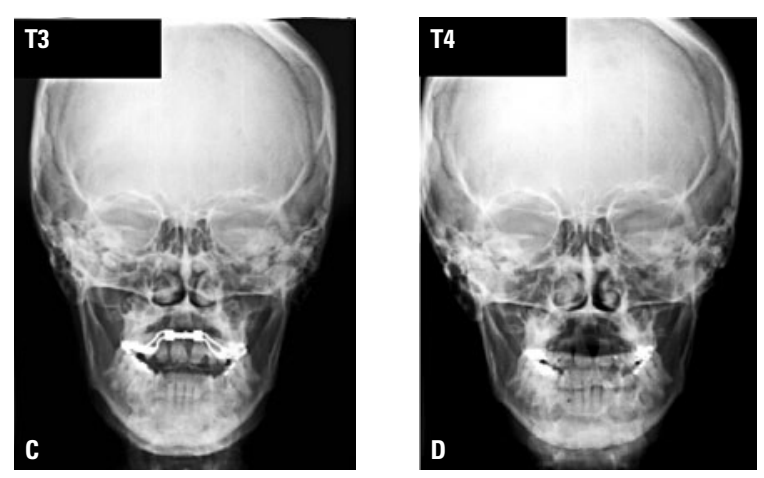

\section{Tratamento ortodôntico-cirúrgico}

\section{Aparelho expansor}

Para cada paciente foi confeccionado o expansor maxilar tipo Hyrax, com o parafuso expansor (Morelli ${ }^{\circledast}$, Sorocaba/SP) com capacidade para $13 \mathrm{~mm}$ de expansão. Foram utilizados quatro dentes de ancoragem, sendo os dois primeiros molares e os dois primeiros pré-molares (Fig. 2). Todos os aparelhos foram confeccionados de forma padronizada no laboratório do Programa de Pós-graduação em Odontologia da Universidade Metodista de São Paulo, por um mesmo técnico laboratorial. Os fios ortodônticos de $1,2 \mathrm{~mm}$ de diâmetro fizeram as conexões entre as bandas nas superfícies vestibular e palatina. Utilizou-se solda de prata na união entre os fios e as bandas e entre as bandas e os parafusos. Esses aparelhos foram instalados

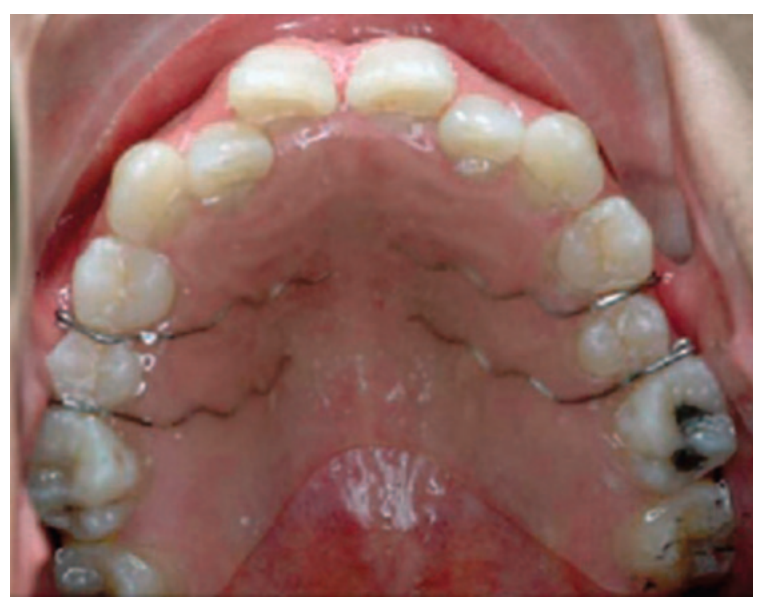

FIGURA 3 - Placa removível de acrílico. 
uma semana antes da realização da cirurgia, após prévia separação com elástico separador, com cimento de ionômero de vidro.

\section{Técnica cirúrgica}

Todas as cirurgias foram realizadas na Divisão de Cirurgia Plástica e Queimados do Hospital das Clínicas da Faculdade de Medicina da Universidade de São Paulo, por um mesmo cirurgião.

A técnica cirúrgica consistiu de uma osteotomia de maxila tipo Le Fort I modificada, caracterizada pela osteotomia sagital mediana da maxila e não-abordagem da sutura pterigopalatina ${ }^{13}$.

O dispositivo expansor foi, então, ativado de forma que os maxilares fossem expandidos cerca de 3 a $5 \mathrm{~mm}$ antes do fechamento dos tecidos incisados, para conferência das osteotomias e avaliação da simetria da expansão bilateral. Caso fosse verificada apenas a expansão unilateral, as osteotomias seriam conferidas e complementadas no lado estável, para evitar uma expansão unilateral assimétrica. Posteriormente, o parafuso expansor foi desativado, sendo mantido com uma abertura de $1 \mathrm{~mm}$, e realizado o fechamento por planos das incisões, com pontos separados de vicryl 3-0.

\section{Ativação e contenção do parafuso expansor}

O início da ativação do parafuso expansor para a obtenção da expansão rápida da maxila ocorreu a partir do terceiro dia após a realização da cirurgia. Na primeira consulta foi realizada uma ativação (1/4 de volta). Após as orientações, o responsável pela ativação do aparelho foi instruído a realizar 1/4 de volta a cada 12 horas, totalizando 2/4 de volta ao dia, sendo a chave inserida e direcionada de anterior para posterior ${ }^{8}$. Os pacientes retornavam uma vez por semana para controle da ativação, até completar a abertura total do parafuso ou alcançar os objetivos oclusais.

Os limites para a expansão foram determinados por critérios eminentemente clínicos, sempre procurando realizar uma sobrecorreção dentária, com o objetivo de maximizar o efeito ortopédico, e permitir a subsequente verticalização dos dentes inclinados e a remodelação do processo alveolar. A pausa da ativação do parafuso ocorria quando havia o contato das vertentes vestibulares das cúspides palatinas dos molares superiores com as vertentes linguais das cúspides vestibulares dos molares inferiores ${ }^{8}$.

Passada a fase de expansão, o parafuso expansor foi estabilizado com um fio de latão, evitando a sua desativação durante a fase de contenção ${ }^{14,20}$. Três meses após essa estabilização, o disjuntor tipo Hyrax foi removido e, imediatamente após, foi instalada uma placa removível de contenção, utilizada por mais três meses (Fig. 3).

\section{Traçado cefalométrico}

Para efeito de padronização, as radiografias foram obtidas de acordo com as normas preconizadas pelo Programa de Pós-graduação em Odontologia - área de concentração em Ortodontia - da Universidade Metodista de São Paulo, por um mesmo profissional técnico em Radiologia.

Para a realização dos traçados cefalométricos, as telerradiografias foram digitalizadas em escala de tons de cinza, com resolução de 300dpi e tamanho da imagem em 100\%, no formato TIFF, as quais posteriormente foram importadas pelo software Radiocef Studio 2, da Empresa Radio Memory Ltda, Belo Horizonte / MG.

As estruturas anatômicas, os pontos cefalométricos e as variáveis lineares utilizados estão apresentados na figura 4, no quadro 1 e na figura 5, respectivamente, e a figura 6 apresenta a tela do programa Radiocef Studio 2 com o respectivo traçado cefalométrico computadorizado.

As variáveis lineares medidas foram (Fig. 5):

A) Largura facial: distância, em milímetros, determinada entre os pontos ZA e AZ.

B) Largura nasal: distância, em milímetros, determinada entre os pontos NC e CN.

C) Largura maxilar: distância, em milímetros, determinada entre os pontos JL e JR.

D) Distância intermolares superiores: distância, 


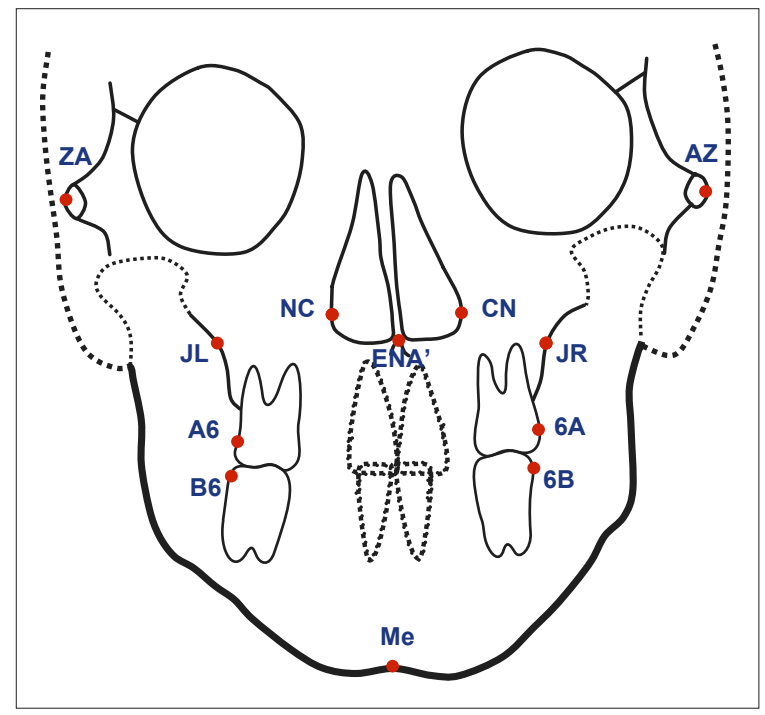

FIGURA 4 - Desenho anatômico e pontos cefalométricos.

\begin{tabular}{|c|c|c|}
\hline LOCALIZAÇÃO & SIGLA & DEFINIÇÃo \\
\hline $\begin{array}{l}\text { Ponto zigomático- } \\
\text { mediano esquerdo } \\
\text { Ponto zigomático- } \\
\text { mediano direito }\end{array}$ & ZA & $\begin{array}{l}\text { Ponto mediano situado no } \\
\text { centro do bordo externo da } \\
\text { apófise zigomática }\end{array}$ \\
\hline $\begin{array}{l}\text { Ponto Nasal esquerdo } \\
\text { Ponto Nasal direito }\end{array}$ & $\begin{array}{l}\mathrm{NC} \\
\mathrm{CN}\end{array}$ & $\begin{array}{l}\text { Ponto mais externo do con- } \\
\text { torno da cavidade nasal }\end{array}$ \\
\hline Ponto Jugal esquerdo & $\mathrm{JL}$ & $\begin{array}{l}\text { Ponto mais côncavo da } \\
\text { tuberosidade maxilar, na } \\
\text { interseção do seu contorno } \\
\text { com a apófise zigomática }\end{array}$ \\
\hline $\begin{array}{l}\text { Ponto molar superior } \\
\text { esquerdo } \\
\text { Ponto molar superior } \\
\text { direito }\end{array}$ & $6 \mathrm{~A}$ & $\begin{array}{c}\text { Ponto mais vestibular da } \\
\text { coroa do primeiro molar } \\
\text { superior }\end{array}$ \\
\hline $\begin{array}{l}\text { Ponto molar inferior } \\
\text { esquerdo } \\
\text { Ponto molar inferior } \\
\text { direito }\end{array}$ & B6 & $\begin{array}{c}\text { Ponto mais vestibular da } \\
\text { coroa do primeiro molar } \\
\text { inferior }\end{array}$ \\
\hline Ponto Mentoniano & $\mathrm{Me}$ & $\begin{array}{l}\text { Ponto mediano situado } \\
\text { sobre o bordo inferior da } \\
\text { sínfise, abaixo da protube- } \\
\text { rância mentoniana }\end{array}$ \\
\hline $\begin{array}{c}\text { Espinha Nasal } \\
\text { Anterior modificada }\end{array}$ & ENA' & $\begin{array}{c}\text { Ponto mediano acima da } \\
\text { Espinha Nasal Anterior } \\
\text { situado na junção entre as } \\
\text { cavidades nasais direita e } \\
\text { esquerda }\end{array}$ \\
\hline
\end{tabular}

QUADRO 1 - Descrição dos pontos cefalométricos utilizados na elaboração do cefalograma em norma frontal.

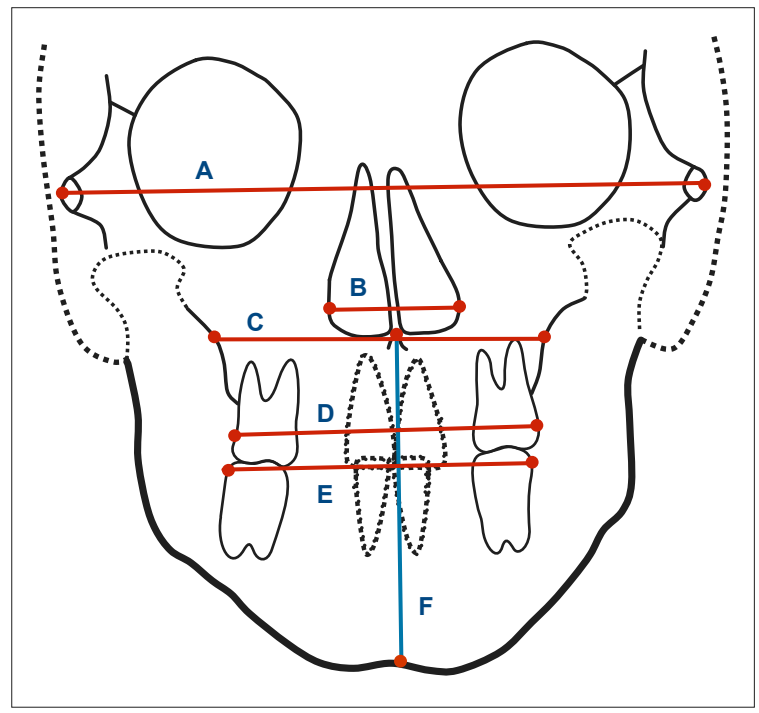

FIGURA 5 - Variáveis lineares.

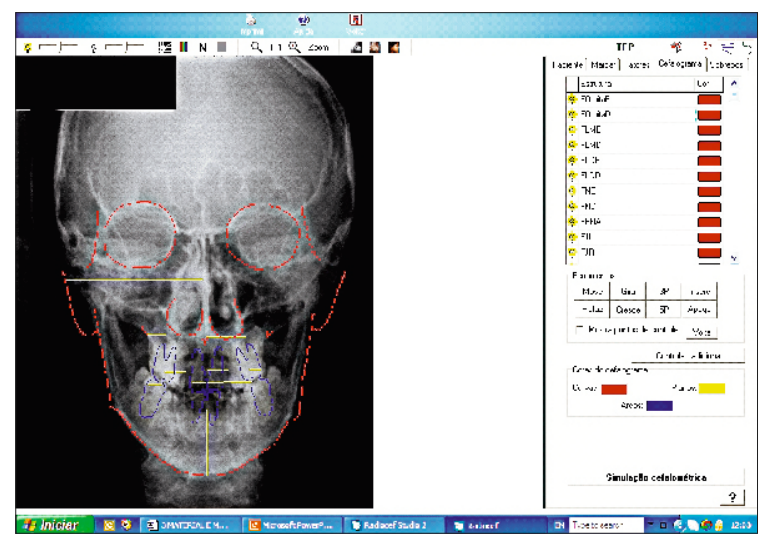

FIGURA 6 - Tela do programa Radiocef Studio 2 com respectivo traçado cefalométrico computadorizado.

em milímetros, determinada entre os pontos A6 e $6 \mathrm{~A}$.

E) Distância intermolares inferiores: distância, em milímetros, determinada entre os pontos B6 e $6 \mathrm{~B}$.

F) Altura facial anteroinferior (AFAI): distância, em milímetros, determinada entre os pontos ENA' e Me. 
Análise estatística

Para a obtenção do erro intraexaminador, foram retraçadas e medidas novamente as radiografias de oito pacientes, selecionados aleatoriamente, após um período mínimo de dois meses. Foram utilizadas as radiografias nas quatro fases, totalizando 32 radiografias.

Aplicou-se o teste t pareado (variáveis dependentes) para a verificação do erro sistemático. $\mathrm{Na}$ determinação do erro casual, utilizou-se o cálculo de erro proposto por Dahlberg. Em todos os testes adotou-se nível de significância de $5 \%^{32}$.

A confiabilidade dos traçados cefalométricos, medidas e resultados foi submetida à avaliação da metodologia, para a obtenção do erro do método. Embora para a obtenção do erro do método deva-se retraçar 20\% do total das radiografias
(12 radiografias), por se tratar de uma amostra reduzida, optamos por retraçar novamente 53\% (32 radiografias nas fases pré-expansão, pós-expansão imediata, pós-expansão de três meses e de seis meses), escolhidas aleatoriamente, após um período de dois meses, para a verificação do erro intraexaminador. Aplicou-se o teste " $\mathrm{t}$ " pareado para o erro sistemático e a fórmula de Dahlberg para estimar as grandezas dos erros casuais.

\section{RESULTADOS}

Os resultados são apresentados nas tabelas 1 , 2 e 3.

\section{DISCUSSÃO}

Desde os primeiros trabalhos publicados, em 1860, por Angell1,2, a ERM é utilizada para a cor-

TABELA 1 - Média, desvio-padrão das duas medições, teste "t" pareado e erro de Dahlberg para avaliar o erro sistemático e o erro casual.

\begin{tabular}{|c|c|c|c|c|c|c|c|}
\hline \multirow{2}{*}{ medida } & \multicolumn{2}{|c|}{$1^{\text {a }}$ MEDIÇÃO } & \multicolumn{2}{|c|}{$2^{\mathrm{a}}$ MEDIÇÃO } & \multirow[b]{2}{*}{$t$} & \multirow[b]{2}{*}{$\mathbf{P}$} & \multirow{2}{*}{ ERRO CASUAL } \\
\hline & média & d.p. & média & d.p. & & & \\
\hline$Z A-A Z$ & 132,66 & 6,96 & 132,58 & 6,77 & 0,413 & 0,681 n.s. & 1,11 \\
\hline NC-CN & 35,59 & 2,42 & 35,78 & 2,99 & 0,663 & 0,510 n.s. & 1,57 \\
\hline JL-JR & 65,15 & 4,15 & 65,28 & 4,14 & 0,487 & 0,628 n.s. & 1,36 \\
\hline$A 6-6 A$ & 61,45 & 6,42 & 60,58 & 6,04 & 2,241 & $0,029^{*}$ & 2,20 \\
\hline B6-6B & 60,49 & 4,43 & 59,56 & 4,55 & 2,231 & $0,031^{*}$ & 2,03 \\
\hline ENA'-Me & 78,54 & 6,57 & 78,56 & 6,62 & 0,45 & 0,654 n.s. & 0,25 \\
\hline
\end{tabular}

n.s. = diferença estatisticamente não-significativa.

* = diferença estatisticamente significativa $(p<0,05)$.

TABELA 2 - Média, desvio-padrão das seis medidas estudadas nas quatro fases de avaliação, e os resultados da Análise de Variância e Teste de Tukey.

\begin{tabular}{|c|c|c|c|c|c|c|c|c|c|}
\hline \multirow{2}{*}{ medida } & \multicolumn{2}{|c|}{ INICIAL } & \multicolumn{2}{|c|}{ PÓS-EXPANSÃO } & \multicolumn{2}{|c|}{3 MESES } & \multicolumn{2}{|c|}{6 MESES } & \multirow{2}{*}{$\mathbf{P}$} \\
\hline & média & d.p. & média & d.p. & média & d.p. & média & d.p. & \\
\hline$Z A-A Z$ & 132,60 & 7,22 & 132,83 & 7,07 & 132,32 & 7,00 & 132,90 & 7,27 & 0,315 n.s. \\
\hline $\mathrm{NC}-\mathrm{CN}$ & $34,09^{a}$ & 2,68 & $36,39^{b}$ & 2,08 & $35,56^{b}$ & 2,03 & $36,32^{b}$ & 2,31 & $<0,001^{*}$ \\
\hline JL-JR & $62,15^{\mathrm{a}}$ & 3,85 & $66,44^{\mathrm{b}}$ & 3,85 & $66,34^{b}$ & 4,19 & $65,68^{b}$ & 3,49 & $<0,001^{*}$ \\
\hline$A 6-6 A$ & $56,27^{a}$ & 5,57 & $63,93^{b}$ & 5,71 & $63,45^{b}$ & 6,20 & $62,16^{b}$ & 5,58 & $<0,001^{*}$ \\
\hline B6-6B & 59,58 & 4,58 & 61,10 & 4,10 & 61,41 & 4,17 & 59,84 & 5,12 & 0,138 n.s. \\
\hline ENA'-Me & $75,68^{a}$ & 6,72 & $80,77^{b}$ & 6,56 & $79,34^{c}$ & 6,23 & $78,37^{c}$ & 6,31 & $<0,001^{*}$ \\
\hline
\end{tabular}

n.s. = diferença estatisticamente não-significativa.

* = diferença estatisticamente significativa $(p<0,05)$.

Fases com a mesma letra não possuem diferença estatisticamente significativa entre si (teste de Tukey). 
TABELA 3 - Variações das médias estudadas nas quatro fases de avaliação.

\begin{tabular}{cccccccccc} 
medida & \multicolumn{2}{c}{ INICIAL X PóS-EXPANSÃO } & \multicolumn{2}{c}{ PóS-EXPANSÃO X 3 MESES } & \multicolumn{2}{c}{3 MESES X 6 MESES } & \multicolumn{3}{c}{ INICIAL X 6 MESES } \\
& média & d.p. & média & d.p. & média & d.p. & média & d.p. \\
ZA-AZ & 0,23 & 0,99 & $-0,51$ & 1,61 & 0,58 & 1,71 & 0,30 & 0,33 \\
NC-CN & 2,30 & 1,44 & $-0,83$ & 1,07 & 0,76 & 1,02 & 2,23 & 2,39 \\
JL-JR & 4,29 & 2,23 & $-0,10$ & 0,78 & $-0,66$ & 1,66 & 3,53 & 3,45 \\
A6-6A & 7,66 & 4,19 & $-0,48$ & 1,05 & $-1,29$ & 2,53 & 5,89 & 5,74 \\
B6-6B & 1,52 & 2,73 & 0,31 & 1,88 & $-1,57$ & 2,80 & 0,26 & 0,02 \\
\hline ENA'-Me & 5,09 & 2,50 & $-1,43$ & 1,13 & $-0,97$ & 0,78 & 2,69 & 2,02 \\
\hline
\end{tabular}

reção da atresia maxilar com a separação da sutura palatina mediana, possível em pacientes jovens devido à menor resistência óssea. Entretanto, à medida que a maturidade esquelética aumenta, o prognóstico torna-se duvidoso e, por vezes, exigirá um auxílio cirúrgico ${ }^{3,8,18,24}$. Apesar do efeito esquelético alcançado, a literatura demonstra uma inclinação vestibular excessiva dos dentes posteriores que, com o aumento da maturidade esquelética, torna-se mais evidente e, portanto, osteotomias deverão ser realizadas, prevenindo danos ao periodonto ${ }^{9,10,17,18,28,29}$.

Após a terceira década de vida, a obliteração sutural alcança estágios de resistência praticamente impossíveis de serem vencidos pela ação ortopédica isolada, e uma combinação entre Cirurgia e Ortodontia deverá ser indicada, diminuindo a resistência dos pilares ósseos (suturas palatina mediana, nasomaxilar, pterigopalatina e zigomatomaxilar) 4,8,11,16,19,20,23,24,25. Para facilitar a expansão maxilar, Brown (1938, apud CAPELOZZA FILHO et $\mathrm{al}^{8}{ }^{8}$ ) descreveu uma técnica cirúrgica para abordagem da sutura palatina mediana, que era considerada a área de maior resistência. Desde então, diversas osteotomias foram idealizadas na tentativa de garantir o efeito ortopédico da ERMAC, entretanto, na literatura não existe um consenso sobre qual seria a mais apropriada, o que torna o assunto polêmico. Para alguns, a osteotomia deve garantir êxito na expansão, porém, com um procedimento conservador. $\mathrm{O}$ presente estudo baseou-se na osteotomia maxilar tipo Le Fort I, caracterizada pela não-abordagem do processo pterigoide do esfenoide, associada à osteotomia palatina mediana. Alguns autores realizaram essa técnica preservando o processo pterigoide do esfenoide, com resultados que demonstraram sucesso na expansão e na estabilidade ${ }^{8,24}$. Entretanto, para outros, esse procedimento só é válido para as atresias que possuam maior severidade nas regiões anterior e média da maxila, e a abordagem posterior deverá ser realizada quando houver a indicação da expansão posterior ${ }^{4,5,7,13,20}$.

O aparelho expansor preconizado para a ERMAC apresentou algumas mudanças daquele proposto por Haas ${ }^{17,18}$. Biederman ${ }^{6}$, em 1968, desenvolveu o Hyrax, um aparelho dentossuportado que facilita a higienização, diminui os riscos de traumas e ulcerações no palato, além de os resultados dentoesqueléticos não apresentarem diferenças na expansão final21,28 - o qual, por esses motivos, foi escolhido para o presente estudo.

Com o intuito de avaliar as alterações decorrentes da ERMAC, as telerradiografias em norma frontal foram escolhidas por serem um método confiável e eficiente para o correto diagnóstico e avaliação dos resultados da disjunção. Entretanto, essas apresentaram desvantagens relacionadas à identificação e marcação de pontos de referência estáveis e à sobreposição das estruturas craniofaciais, dificultando a delimitação anatômica e a obtenção das medidas cefalométricas. A análise cefalométrica empregada baseou-se no traçado descrito por Ricketts ${ }^{26}$, o qual possibilita observar o comportamento ortopédico 
transversal e vertical, corroborando com os trabalhos de outros autores ${ }^{12,14,15,21}$.

Para a elaboração dos traçados cefalométricos e mensuração das variáveis descritas, utilizou-se o programa computadorizado Radiocef studio 2, que demonstrou ser um método confiável e muito utilizado no meio científico na elaboração de pesquisas $^{14}$.

A confiabilidade dos traçados cefalométricos, medidas e resultados foi submetida à avaliação da metodologia para a obtenção do erro do método. Por se tratar de uma amostra reduzida, optou-se por retraçar 53\% dos traçados (32 radiografias nas fases pré-expansão, pós-expansão imediata, pós-expansão de três meses e de seis meses), escolhidos aleatoriamente, após um período de dois meses, para a verificação do erro intraexaminador. Aplicou-se o teste " $t$ " pareado para o erro sistemático e a fórmula de Dahlberg para estimar as grandezas dos erros casuais.

Apesar da tabela 1 apresentar duas variáveis, das seis estudadas, com erro sistemático e também com erro casual elevado, isso se justificou devido às mesmas (A6-6A e B6-6B) apresentarem um grau de dificuldade muito grande de visualização e, consequentemente, de marcação dos pontos ${ }^{12,15}$. Apesar disso, as médias das duas medições foram bem próximas, o que validou a utilização dessas variáveis para as comparações posteriores.

\section{Largura facial (ZA-AZ)}

Pela análise das tabelas 2 e 3, pode-se observar os valores médios e as alterações médias em todas as fases estudadas. Apesar de pequenas variações entre as fases (aumento de $0,23 \mathrm{~mm}$ de $\mathrm{Tl}$ para T2; diminuição de $0,51 \mathrm{~mm}$ de T2 para T3; aumento de $0,58 \mathrm{~mm}$ de T3 para T4; aumento de $0,30 \mathrm{~mm}$ de $\mathrm{Tl}$ para T4), não existiu diferença estatisticamente significativa quando foi realizada a análise de variância.

Esses resultados são concordantes com os estudos de Berger et al. ${ }^{4}$, que compararam a estabilidade da ERMAC com a da ERM ortopédica, por meio de modelos e telerradiografias em norma frontal, e encontraram um aumento de $0,53 \mathrm{~mm}$ pós-expansão imediata; de $0,16 \mathrm{~mm}$ no período de contenção antes da remoção do expansor; e de 0,47 mm após um ano com placa de contenção.

Massulo et al. $^{20}$ analisaram, por meio de telerradiografias em norma frontal, as alterações promovidas pela ERMAC e não encontraram modificações significativas. Esses resultados corroboraram os achados de Gondo ${ }^{14}$, que avaliou as alterações dentárias e esqueléticas da ERMAC por meio de telerradiografias em norma frontal - em uma amostra de 17 pacientes adultos, nas fases pré-expansão (Tl), imediatamente após a expansão (T2) e após três meses (T3) - e encontrou uma redução de $0,17 \mathrm{~mm}$ de $\mathrm{Tl}$ para $\mathrm{T} 2,0,58 \mathrm{~mm}$ de T2 para T3, e 0,74mm de T1 para T3. Esses resultados demonstraram que, apesar da pouca ou nenhuma alteração da projeção zigomática após a ERMAC, não existiu diferença estatisticamente significativa.

\section{Largura nasal (NC-CN)}

Para a realização da ERMAC, há necessidade de liberar as zonas de resistência da face, desarticulando algumas suturas, como a zigomatomaxilar, a pterigopalatina e a nasomaxilar. Por essa razão, observa-se que, após os procedimentos de disjunção, ocorre um aumento na cavidade na-

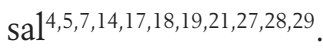

Para observar esse efeito na amostra estudada, verificou-se que, pela análise de variância (Tab. 2, $3)$, houve diferença estatisticamente significativa entre as fases. Para a verificação das fases que mostraram essa diferença, utilizou-se o teste de Tukey, que revelou a existência de diferença estatisticamente significativa entre T1 e T2, T1 e T3, e T1 e T4, devido ao aumento da cavidade nasal decorrente da abertura da sutura palatina mediana após a ERMAC.

Os resultados do presente estudo, nas fases avaliadas, apontam para um aumento estatisticamente significativo de $2,30 \mathrm{~mm}$, em média, da cavidade 
nasal entre as fases T1 e T2 (Tab. 3), concordando com os estudos de Berger et al. ${ }^{4}$, que compararam - numa amostra de 52 pacientes (28 cirúrgicos e 24 não-cirúrgicos) - a estabilidade da ERMAC com a da ERM ortopédica, por meio de modelos (distâncias intercaninos e intermolares) e telerradiografias em norma frontal (larguras alveolar, maxilar, zigomática e nasal), e encontraram um aumento de $1,72 \mathrm{~mm}$ pós-expansão imediata. Massulo et al. ${ }^{20}$ encontraram um aumento de $1,81 \mathrm{~mm}$ e Gondo ${ }^{14}$ encontrou um aumento de $1,18 \mathrm{~mm}$.

$\mathrm{Na}$ fase T3, ou seja, três meses pós-expansão (com o próprio disjuntor como contenção), a largura nasal apresentou uma pequena diminuição, de $0,83 \mathrm{~mm}$, quando comparada à de $\mathrm{T} 2$, porém não demonstrou diferença estatística, indicando estabilidade do resultado. Esses resultados corroboram os dos estudos de Massulo et al. ${ }^{20}$ e Gon$\mathrm{do}^{14}$, que também observaram estabilidade na fase de três meses pós-expansão. Esses achados também foram observados por Berger et al. ${ }^{4}$, em um tempo de observação semelhante, ou seja, a largura nasal diminuiu $0,41 \mathrm{~mm}$, sem diferença estatisticamente significativa.

Na fase T4, de seis meses pós-expansão (com placa removível de acrílico como contenção), quando comparada com $\mathrm{T} 3$, houve um aumento de $0,76 \mathrm{~mm}$ em média, entretanto, estatisticamente não-significativo. Esse resultado foi concordante com os de Berger et al. ${ }^{4}$, que acompanharam um ano pós-expansão, com placa de Hawley como contenção, e encontraram um aumento de $0,14 \mathrm{~mm}$.

A análise final dessa variável demonstra que houve um aumento da cavidade nasal decorrente da ERMAC imediatamente após a expansão e esse se manteve estável após seis meses de avaliação.

\section{Largura maxilar (JL-JR)}

A variável largura maxilar é muito importante para definir o diagnóstico da atresia maxilar e avaliar as alterações promovidas pela ERMAC. Os valores médios da largura maxilar pré-expansão
(T1) encontrados neste estudo foram de $62,15 \mathrm{~mm}$ (Tab. 2), indicando uma deficiência transversal ${ }^{26,27}$. Imediatamente após a ERMAC (T2), observou-se que houve um aumento estatisticamente significativo de $4,29 \mathrm{~mm}$, muito semelhante ao dos estudos de Berger et al. ${ }^{4}$, que avaliaram a estabilidade da ERMAC realizada com osteotomia tipo Le Fort I e da ERM ortopédica, por meio de telerradiografias em norma frontal - pós-expansão imediata (T1); três meses pós-expansão (T2); e um ano pós-expansão, com placa de Hawley como contenção (T3) - e evidenciaram um aumento, em média, de $3,54 \mathrm{~mm}$ para o grupo ortopédico e de $3 \mathrm{~mm}$ para o cirúrgico, fato que pode ser explicado pelo crescimento interalveolar dos pacientes adolescentes que compuseram a amostra.

Massulo et al. ${ }^{20}$ analisaram a ERMAC em pacientes com média de idades de 24 anos, submetidos à osteotomia Le Fort I subtotal (caracterizada pela abordagem do processo pterigoide do esfenoide e sutura palatina mediana) - nas fases pósexpansão imediata (T1), três meses pós-expansão com o próprio expansor como contenção (T2), e três meses de contenção (T3) - e encontraram um aumento transversal maxilar de 2,91 mm.

Entretanto, Byloff e Mossaz ${ }^{7}$ avaliaram, por meio de telerradiografias em norma frontal, 14 pacientes adultos - que apresentavam uma atresia maxilar maior do que $5 \mathrm{~mm}$ e foram submetidos à osteotomia vestibular da abertura piriforme ao processo pterigoide do esfenoide e da sutura palatina mediana - e encontraram um aumento após a ERMAC de 2,62mm. Gondo ${ }^{14}$ - que analisou a ERMAC realizada com osteotomia tipo Le Fort I com abordagem da sutura palatina mediana e não-liberação do processo pterigoide do esfenoide - encontrou um aumento de 4,13mm.

Outros trabalhos apresentaram resultados semelhantes para essa variável, entretanto, utilizando expansão rápida realizada sem auxílio cirúrgico $^{10,17,18,21,27,28,29}$.

$\mathrm{Na}$ fase de três meses pós-expansão (com o próprio disjuntor como contenção) (T3), houve 
uma pequena diminuição de $0,10 \mathrm{~mm}$, observada nas tabelas 2 e 3 , porém, não-significativa estatisticamente. Os resultados foram concordantes com os dos trabalhos de Berger et al. ${ }^{4}$; Massulo et al. ${ }^{20}$; Byloff, Mossaz e Gondo ${ }^{14}$, que encontraram diferenças de $0,13 \mathrm{~mm}, 0,07 \mathrm{~mm}, 0,50 \mathrm{~mm}$ e $0,30 \mathrm{~mm}$, respectivamente; porém, também não demonstrando significância estatística.

A avaliação da fase de seis meses pós-expansão com a placa removível de acrílico demonstrou que houve uma pequena diminuição de $0,66 \mathrm{~mm}$ (Tab. 2 , 3), entretanto, pelo método estatístico empregado, essa não representou significância, corroborando o estudo de Berger et $\mathrm{al}^{4}{ }^{4}$, que apresentou, no período de contenção com placa de Hawley, um decréscimo de $0,67 \mathrm{~mm}$, em média.

\section{Distância intermolares superiores (A6-6A)}

Pela análise de variância (Tab. 2, 3), houve diferença estatisticamente significativa nas quatro fases do tratamento. Para a verificação das fases que mostraram essa diferença, utilizou-se o teste de Tukey, que revelou a existência de diferença estatisticamente significativa entre $\mathrm{Tl}$ e $\mathrm{T} 2$, $\mathrm{T} 1$ e T3, e T1 e T4, devido ao aumento da distância intermolares superiores após a ERMAC.

A análise dos valores médios nas fases estudadas está disposta na tabela 2. Imediatamente após a ERMAC (T2), observou-se um aumento da distância intermolares superiores, demonstrando que as coroas estão mais vestibularizadas, em média, 7,66mm (Tab. 3). Esses resultados são concordantes com os do estudo de Berger et al. ${ }^{4}$, que observaram um aumento de $4,29 \mathrm{~mm}$ e $3,42 \mathrm{~mm}$, para a ERM e a ERMAC, respectivamente, sugerindo que há maior inclinação vestibular nos pacientes submetidos à ERM, devido à maior resistência óssea apresentada pelas suturas craniofaciais.

Em 1992, Pogrel et al. ${ }^{24}$ analisaram a ERMAC em pacientes com média de idades de 23 anos, com osteotomias laterais sem incluir a abertura piriforme e a fossa pterigopalatina, portanto, mais conservadoras. Após três semanas do término da ativação, apresentaram uma média de expansão intermolares superiores de $7,5 \mathrm{~mm}$.

Também em 1992, Mossaz, Byloff e Richter ${ }^{22}$ demonstraram a ERMAC em quatro casos clínicos, dois com osteotomias unilaterais e dois com bilaterais, compreendendo da abertura piriforme ao processo pterigoide do esfenoide. $\mathrm{Na}$ ERMAC bilateral, encontraram aumento da distância intermolares superiores de $9,8 \mathrm{~mm}$ e $10 \mathrm{~mm}$, o qual nos dois casos unilaterais foi de $9,5 \mathrm{~mm}$ e $7,7 \mathrm{~mm}$.

Strömberg e Holm ${ }^{30}$ selecionaram 20 pacientes com média de idades de 36 anos, submetidos a osteotomias bilaterais tipo Le Fort I, analisados por meio de telerradiografias em normas lateral e frontal e modelos de estudo - pré-expansão (T1), após a remoção do expansor (T2), e após o término do tratamento ortodôntico (T3) - com acompanhamento de três anos e seis meses, e observaram uma expansão de $7,1 \mathrm{~mm}$. Byloff e Mossaz ${ }^{7}$ realizaram a ERMAC em 14 pacientes adultos, com osteotomias vestibulares e separação do processo pterigoide do esfenoide, e observaram um aumento pós-expansão (T2) e recidiva no período de contenção. Para Gondo ${ }^{14}$, após a ERMAC, houve um aumento médio da distância intermolares superiores de $10,23 \mathrm{~mm}$.

No presente estudo, na fase de três meses pósexpansão, com o próprio disjuntor como contenção (T3), foi detectada uma pequena diminuição, estatisticamente insignificante (Tab. 2, 3). Esse resultado foi discordante dos encontrados por Berger et al. ${ }^{4}$, Massulo et $\mathrm{al}^{20}$ e Gondo ${ }^{14}$, que encontraram um pequeno aumento decorrente de eventuais forças residuais, porém, estatisticamente insignificante. Outros autores ${ }^{16,17,18,21,27,28,29}$ apresentaram resultados semelhantes para essa variável, entretanto, a expansão rápida foi realizada sem auxílio cirúrgico.

$\mathrm{Na}$ última fase avaliada, seis meses pós-expansão com placa removível de acrílico (T4), encontrou-se aumento estatisticamente significativo, quando comparado ao da fase pré-expansão (T1) (Tab. 2, 3). Entretanto, apesar da recidiva média 
de $1,29 \mathrm{~mm}$, quando comparada com a fase T3, não apresentou diferença estatisticamente significativa, demonstrando estabilidade. Pogrel et al. ${ }^{24}$ observaram que a ERMAC em pacientes adultos, apesar das osteotomias mais conservadoras, manteve as distâncias intermolares superiores estáveis, com pequena recidiva média de $0,88 \mathrm{~mm}$ um ano após a ERMAC. Mossaz, Byloff e Richter ${ }^{22}$ também observaram que, na ERMAC bilateral, houve uma diminuição de $0,2 \mathrm{~mm}$ e $0,6 \mathrm{~mm}$ no final da contenção, e $0,5 \mathrm{~mm}$ e $3,7 \mathrm{~mm}$ um ano após o final da contenção, enquanto, para a ERMAC unilateral, $3,6 \mathrm{~mm}$ e $2,5 \mathrm{~mm}$ no final da contenção, e $1,5 \mathrm{~mm}$ e $1,7 \mathrm{~mm}$ um ano após o final da contenção, o que não representou significância clínica. Strömberg e Holm $^{30}$ observaram que, após a expansão imediata, houve um aumento de $7,1 \mathrm{~mm}$, sendo que, no acompanhamento de três anos e seis meses após o término do tratamento ortodôntico, ocorreu uma recidiva média de $1,2 \mathrm{~mm}$.

\section{Distância intermolares inferiores (B6-6B)}

Apesar do pequeno aumento de $1,52 \mathrm{~mm}$ pósexpansão imediata (Tab. 2, 3), a ERMAC não demonstrou ser efetiva para promover alterações significativas da variável distância intermolares inferiores. Entretanto, a distância intermolares inferiores, mesmo essa não sendo a região de ancoragem $^{14,17,21,28,29}$, aumentou devido ao contato das cúspides palatinas dos molares superiores com as cúspides vestibulares dos molares inferiores. Essa situação promove mudanças na resultante de forças, e as alterações musculares da face e da língua proporcionaram uma expansão espontânea.

\section{Altura facial anteroinferior (ENA'- Me)}

$\mathrm{Na}$ determinação dessa variável foram encontradas dificuldades para a visualização da estrutura anatômica e, consequentemente, para a marcação do ponto Espinha Nasal Anterior (ENA), principalmente na fase pós-expansão cirúrgica. Por essa razão, foi utilizado o ponto ENA', localizado mais superiormente, entre as paredes mediais das cavi- dades nasais direita e esquerda, o qual nos pareceu ser mais confiável.

A ERM, com ou sem assistência cirúrgica, estabelece muitos benefícios, como: descruzamento da mordida; alteração na forma do arco; recuperação de espaço - o que pode evitar, em algumas situações, as extrações dentárias -; aumento da cavidade nasal; proporciona um melhor desenvolvimento transversal da face; e favorece as correções anteroposteriores. Devido ao deslocamento inferior da maxila, vestibularizações dos dentes de ancoragem e inclinações alveolares, a ERM e a ERMAC produzem alguns efeitos indesejados. A abertura da mordida, a alteração do eixo "Y" de crescimento e a rotação do plano mandibular no sentido horário demonstraram ser desfavoráveis para os pacientes com crescimento vertical9,10,16,18,28,29. Entretanto, Velásquez, Benito e Bravo ${ }^{31}$ analisaram a ERM em 30 pacientes - com média de idades de 12,1 anos, nas fases pré-tratamento (T1) e pós-tratamento ortodôntico (T2), com duração média de 3,1 anos - e observaram, por meio da cefalometria de Ricketts, pequena diferença na comparação entre as duas fases $\left(47,2^{\circ}\right.$ e $47,4^{\circ}$, respectivamente), demonstrando que a altura facial, embora tenha um aumento pós-expansão imediata, após o término do tratamento não demonstrou diferença estatisticamente significativa quando comparada com a altura facial inicial. A alteração vertical da face após a ERMAC, representada pela altura facial anteroinferior (ENA'- Me), pode ser observada nas tabelas 2 e 3 , que demonstram os valores médios nas fases analisadas.

Os resultados pós-expansão imediata (T2) demonstraram aumento médio de $5,09 \mathrm{~mm}$, estatisticamente significativo quando comparado com $\mathrm{T} 1$, o que concorda com outros trabalhos ${ }^{9,17,18,28,29}$, os quais afirmaram ser essa alteração decorrente dos contatos oclusais, da rotação maxilar inferior e da rotação mandibular no sentido horário.

$\mathrm{Na}$ fase de três meses pós-expansão (T3), observou-se uma tendência de retorno aos valores iniciais, com diminuição de 1,43mm, em média, 
demonstrado nas tabelas 2 e 3 , corroborando os estudos de Mazzieiro, Henriques e Freitas ${ }^{21}$.

$\mathrm{Na}$ fase pós-expansão de seis meses, com placa removível de acrílico como contenção (T4), observou-se, mais uma vez, uma tendência de recidiva, média de $0,97 \mathrm{~mm}$, devido ao deslocamento antihorário mandibular e à extrusão e lingualização dos incisivos superiores, entretanto apresentando diferença estatisticamente não-significativa. Esses resultados vão de encontro aos achados de Mazzieiro, Henriques, Freitas ${ }^{21}$; Siqueira, Almeida e Henriques $^{28,29}$.

\section{CONCLUSÃO}

Com base na amostra estudada, na metodologia empregada e nos resultados obtidos, é possível concluir que:

a) Houve um aumento da distância intermolares superiores imediatamente após a expansão, que se manteve estável aos três e aos seis meses pós-expansão. Para a distância intermolares inferiores, a ERMAC não promoveu nenhuma alteração significativa.

b) A largura facial não apresentou alteração após a ERMAC, entretanto, as larguras nasal e maxilar demonstraram um aumento imediatamente após a expansão, que se manteve estável aos três e aos seis meses pós-expansão.

c) A altura facial anteroinferior apresentou um aumento imediatamente após a expansão, com diminuição aos três meses de pós-expansão e estabilidade após seis meses da expansão.

\title{
Cephalometric evaluation of the post surgically assisted rapid maxillary expansion stability
}

\begin{abstract}
Aim: This study evaluated the stability of the dental and skeletal changes produced by the Surgically Assisted Rapid Maxillary Expansion (SARME) in transversal and vertical dimensions. Methods: The sample selected for this retrospective study was comprised by 60 posteroanterior cephalograms, of 15 patients ( 6 males and 9 females), with mean age of 23.3 years. An Hyrax appliance was used and the surgical procedure was characterized by the midsagittal osteotomy and non approach of pterygopalatine suture. The beginning of activation occurred in the third postoperative day, being that the limits for the expansion were determined by eminently clinical criteria. All the patients were radiographed in the following phases: pre-expansion (T1), immediate post-expansion (T2), three months post-expansion (with the Hyrax appliance as retention) (T3), and six months post-expansion (with the acrylic removable plate as retention) (T4). Linear measurements were obtained from the cephalograms tracings generated by a computerized program (Radiocef Studio 2) and statistically analyzed by the variance test (ANOVA) and Tukey at the $5 \%$ level of significance. Results and Conclusion: It was concluded that SARME produced a statistically significant increase of nasal cavity, maxillary width, upper intermolars distance, from T1 to T2, which were maintained in T3 and T4. The facial width and the lower intermolars distance did not presented changes after the SARME. Evaluating the vertical behavior of the face, it was observed an increase of anterior inferior facial height in phases T1 to T2 that decreased after three months of retention (T3) and maintained stable in T4, although increased if compared with T1.
\end{abstract}

Keywords: Maxillary expansion. Maxillofacial surgery. Cephalometrics. 


\section{REFERÊNCIAS}

1. ANGELL, E. H. Treatment of irregularity of the permanent or adult teeth. Part. 1. Dental Cosmos, Philadelphia, v. 1, no. 10, p. 540-544, May 1860.

2. ANGELL, E. H. Treatment of irregularity of the permanent or adult teeth. Part. 2. Dental Cosmos, Philadelphia, v. 1, no. 10, p. 599-600, June 1860.

3. BELL, W. H.; EPKER, B. N. Surgical-orthodontic expansion of the maxilla. Am. J. Orthod., St. Louis, v. 70, no. 5, p. 517-528, Nov. 1976.

4. BERGER, J. L. et al. Stability of orthopedic and surgically assisted rapid palatal expansion over time. Am. J. Orthod. Dentofacial Orthop., St. Louis, v. 114, no. 6, p. 638-645, Dec. 1998.

5. BETTS, N. J. et al. Diagnosis and treatment of transverse maxillary deficiency. Int. J. Adult Orthodon. Orthognath. Surg. Chicago, v. 10, no. 2, p. 75-96, 1995.

6. BIEDERMAN, W. A hygienic appliance for rapid expansion. J. Pract. Orthod., Hempstead, v. 2, no. 2, p. 67-70, Feb. 1968.

7. BYLOFF, F. K.; MOSSAZ, C. F. Skeletal and dental changes following surgically assisted rapid palatal expansion. Eur. J. Orthod., Oxford, v. 26, no. 4, p. 403-409, July/Aug. 2004.

8. CAPELOZZA FILHO, L. et al. Expansão rápida da maxila cirurgicamente assistida. Ortodontia, São Paulo, v. 27, n. 1, p. 21-30, jan./abr. 1994

9. CAPELOZZA FILHO, L.; SILVA FILHO, O. G. Expansão rápida da maxila: considerações gerais e aplicação clínica. Parte I. R. Dental Press Ortodon. Ortop. Facial, Maringá, v. 2, n. 3, p. 88-102, maio/jun. 1997.

10. CAPELOZZA FILHO, L.; SILVA FILHO, O. G. Expansão rápida da maxila: considerações gerais e aplicação clínica. Parte II. R. Dental Press Ortodon. Ortop. Facial, Maringá, v. 2, n. 4, p. 86-108, jul./ago. 1997.

11. EPKER, B. N.; WOLFORD, L. M. Surgical orthodontics expansion of maxilla. In:__. Dento-facial deformity: Surgicalorthodontic correction. St. Louis: C. V. Mosby, 1980. p. 305-331.

12. GIL, C. T. L. de A. Avaliação da precisão na localização dos pontos cefalométricos da análise frontal de Ricketts. 1995. 72 f. Dissertação (Mestrado)-Universidade Estadual Paulista, Faculdade de Odontologia de São José dos Campos, São José dos Campos, 1995

13. GOLDENBERG, D. C. et al. Using computed tomography to evaluate maxillary changes after surgically assisted rapid palatal expansion. J. Craniofac. Surg., Boston, v. 18, no. 2, p. 302-311, Mar. 2007.

14. GONDO, G. Y. Avaliação cefalométrica das alterações transversais e verticais ocorridas em pacientes submetidos à expansão rápida da maxila assistida cirurgicamente. 2006. 149 f. Dissertação (Mestrado em Ortodontia)-Faculdade de Odontologia, Universidade Metodista de São Paulo, São Bernardo do Campo, 2006.

15. GURGEL, J. A.; SANT'ANA, E.; FERREIRA JÚNIOR, O.; SANT'ANA, L. F. M.; GIGLIO, F. P. M. O uso de marcadores para identificação de posicionamento dentário em telerradiografias frontais póstero-anteriores: proposta de um método. R. Dental Press Ortodon. Ortop. Facial, Maringá, v. 10, n. 6 , p. 84-90, nov./dez. 2005.

16. HAAS, A. J. Long-term post treatment evaluation of rapid palatal expansion. Angle Orthod., Appleton, v. 50, no. 3, p. 189-217, July 1980.

17. HAAS, A. J. Rapid expansion of the maxillary dental arch and nasal cavity by opening the midpalatal suture. Angle Orthod., Appleton, v. 31, no. 2, p. 73-90, Apr. 1961.

18. HAAS, A. J. The treatment of maxillary deficiency by opening the midpalatal suture. Angle Orthod., Appleton, v. 35, no. 3, p. 200-217, July 1965.

19. KRAUT, R. A. Surgically assisted rapid maxillary expansion by opening the midpalatal suture. J. Oral Maxillofac. Surg. Philadelphia, v. 42, no. 10, p. 651-655, Oct. 1984.
20. MASSULO, R. C. S. et al. Avaliação cefalométrica lateral de indivíduos submetidos à expansão rápida da maxila assistida cirurgicamente. Rev. Clín. Ortodon. Dental Press, Maringá, v. 5, n. 3, p. 94-105, jun./jul. 2006.

21. MAZZIEIRO, E. T.; HENRIQUES, J. F. C.; FREITAS, M. R. Estudo cefalométrico, em norma frontal, das alterações dentoesqueléticas após a expansão rápida da maxila. Ortodontia, São Paulo, v. 29, n. 1, p. 31-42, jan./abr. 1996.

22. MOSSAZ, C. F.; BYLOFF, F. K.; RICHTER, M. Unilateral and bilateral corticotomies for correction maxillary transverse discrepancies. Eur. J. Orthod., Oxford, v. 14, no. 2, p. 110-116, Apr. 1992.

23. PERSSON, M.; THILANDER, B. Palatal suture closure in man from 15 to 35 years of age. Am. J. Orthod., St. Louis, v. 72, no. 1, p. 42-52, July 1977.

24. POGREL, M. A. et al. Surgically assisted rapid maxillary expansion in adults. Int. J. Adult Orthodon. Orthognath. Surg., Chicago, v. 7, no. 1, p. 37-41, 1992.

25. PROFFIT, W. R.; TURVEY, T. A.; PHILLIPS, C. Orthognathic surgery: A hierarchy of stability. Int. J. Adult Orthodon. Orthognath. Surg., Chicago, v. 11, no. 3, p. 191-204, 1996.

26. RICKETTS, R. M. Perspectives in the clinical application of cephalometrics: The first fifty years. Angle Orthod., Appleton, v. 51, no. 2, p. 115-150, Apr. 1981

27. SATO, K.; VIGORITO, J. W.; CARVALHO, L. S. Avaliação cefalométrica da disjunção rápida da sutura palatina mediana, através da telerradiografia em norma frontal (PA). Rev. Odontol. Metod., São Bernardo do Campo, v. 6, n. 1, p. 123-136, 1985.

28. SIQUEIRA, D. F.; ALMEIDA, R. R.; HENRIQUES, J. F. C. Avaliação cefalométrica em norma frontal, das alterações dentoesqueléticas produzidas pelo aparelho expansor fixo com cobertura de acrílico. R. Dental Press Ortodon. Ortop. Facial, Maringá, v. 7, n. 5, p. 27-44, set./out. 2002.

29. SIQUEIRA, D. F.; ALMEIDA, R. R.; HENRIQUES, J. F. C. Estudo comparativo, por meio de análise cefalométrica em norma frontal, dos efeitos dentoesqueléticos produzidos por três tipos de expansores palatinos. R. Dental Press Ortodon. Ortop. Facial, Maringá, v. 7, n. 6, p. 27-47, nov./dez. 2002.

30. STRÖMBERG, C.; HOLM, J. Surgically assisted rapid maxillary expansion in adults: A retrospective long-term follow-up study. J. Craniomaxillofac. Surg., Edinburgh, v. 23, no. 4, p. 222-227, 1995.

31. VELÁSQUES, P.; BENITO, E.; BRAVO, L. A. Rapid maxillary expansion: A study of the long-term effects. Am. J. Orthod. St. Louis, v. 109, no. 4, p. 361-367, Apr. 1996.

32. ZAR, J. H. Biostatistical analysis. 3rd ed. New Jersey: Prentice-Hall, 1996.
Endereço para correspondência

Pedro Luis Scattaregi

Av. Washington Luiz, 451, Santo Amaro

CEP: 04.662-000 - São Paulo/SP

E-mail: scatta@uol.com.br 